\title{
Empirical analysis of drivers and obstacles for collaborative consumption: A cross-cultural comparison on fashion renting and swapping
}

\author{
Chunmin Lang, Louisiana State University; Su-Kyung Seo, the University of North Carolina at \\ Greensboro; Chuanlan Liu, Louisiana State University
}

Keywords: collaborative consumption, fashion renting, fashion swapping, perceived enjoyment, perceived risks, attitudes, cross culture

Introduction: Over-consumption and a throwaway culture contribute to increased textile waste, which is a growing environmental concern. Approximately 14.3 million tons of textiles now make their way to landfills in the US (US EPA, 2014). Similarly, about 26 million tons of textile waste is generated in China annually, which makes up about $3.5 \%$ to $4 \%$ of the world's waste materials (Xue et al., 2014). Collaborative Consumption (CC), which is based on the shared usage of underutilized or unwanted products, encourages people to focus on product usage rather than ownership (Botsman \& Rogers, 2010). Demailly and Novel (2014) estimate that CC could yield a $20 \%$ reduction in waste. Renting and swapping, two forms of CC (Botsman \& Rogers, 2010), have been suggested as alternative sources of revenue for industry while also extending the life and use frequency of clothing products (WRAP, 2011). Though these concepts of CC are continuing to expand and gain more attention, most are still in their infancy and lack popularity (Demailly \& Novel, 2014). Marketing research conducted by The Nielsen Global Survey (2014) reports that those who are willing to share goods with others amounts to about $49 \%$ in Asia and $18 \%$ in North America.

Previous exploratory studies have discovered some motivations for and obstacles to fashion renting and swapping. However, most of those studies were conducted in Europe and North America. Studies on Chinese consumers' renting and swapping intention and behavior have been neglected. In addition, it has been confirmed that consumers' purchasing behavior, motivation, and product choices are influenced by culture. Given the limited literature on the cross-cultural comparisons of $\mathrm{CC}$ in the textile and apparel industry, this study was designed to empirically identify the motivations and barriers for fashion renting and swapping and to compare the intention towards $\mathrm{CC}$ through renting and swapping between consumers in the US and in China.

Research methods: A convenience sampling strategy was employed with online surveys for data collection in both the United States and China. Data cleaning generated 452 usable samples in the US and 321 usable responses in China. Multi-item scales were used to evaluate the variables, utilizing a 5 -point Likert scale with " $1=$ strongly disagree, to $5=$ strongly agree." Both perceived enjoyment $(\alpha=.965)$ and attitude $(\alpha=.950)$ were measured by five items. Three items were adapted to measure performance risk $(\alpha=.842)$, psychological risk $(\alpha=.829)$ and financial risk $(\alpha=.748)$ respectively. Social risk of $C C(\alpha=.899)$ was measured by four items. In addition, two hypothetical scenario statements were developed for clothing renting and swapping, and participants were requested to rate the level of intention to rent or swap clothing using a 5-point scale. Demographic information was also collected, including gender (US: female $=56.4 \%$, male $=43.6 \%$; China: female $=63.9 \%$, male $=37.1 \%$ ), age (US: $18-35=33.6 \%, 36-50=34.7 \%$, 51$69=31.6 \%$; China: $18-35=67 \%, 36-50=27.7 \%, 51-69=5.3 \%$ ), and education (US: some college $=24.8 \%$, college degree $=38.7 \%$, Master $/$ MBA or higher $=16.6 \%$; China: some college $=20.9 \%$, college degree $=49.2 \%$, Master/MBA or higher $=29.9 \%$ ). The questionnaire was developed in English, which was first translated into Chinese and then was back translated into English to ensure an accurate translation. 
Results: The results of multiple t-tests illustrate significant differences between the two samples in consumers' intentions, perceived enjoyment, attitude, as well as perceived social risk, performance risk and psychological risk (Table 1). In addition, a stepwise regression analysis was conducted to test the influences of independent variables on the intention to rent and swap. The statistical results indicated different factors influencing consumers' intention to rent or swap clothing between the two samples (Table 2).

Table 1. Multiple t-tests

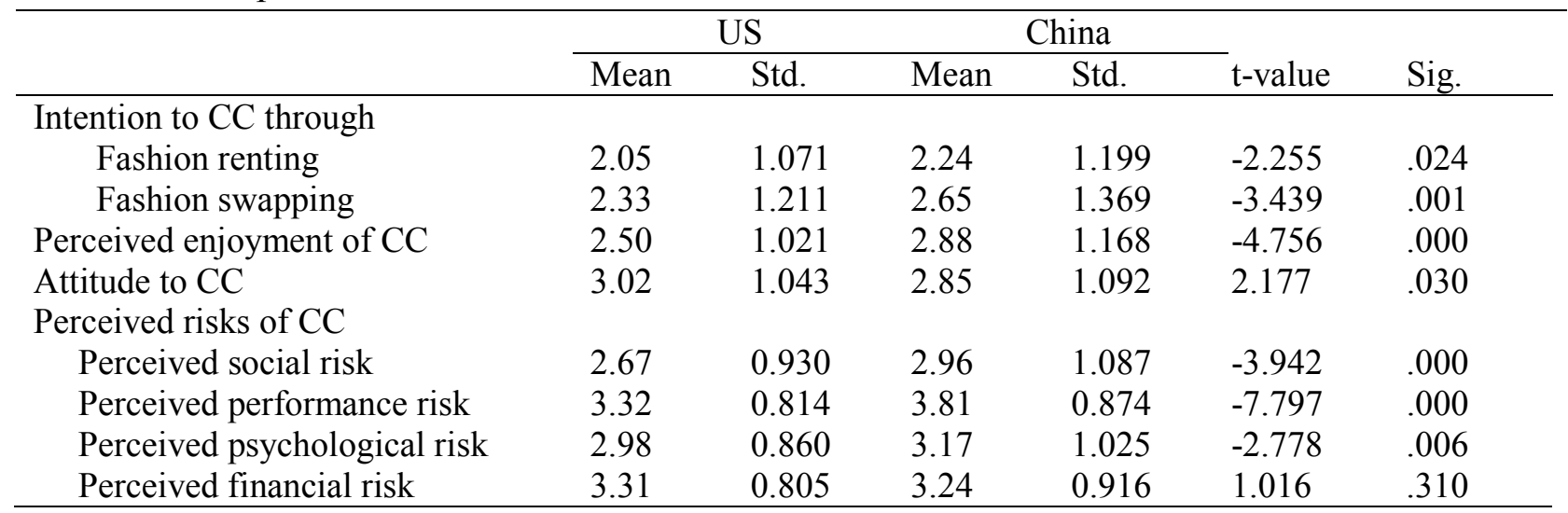

Table 2. Regression analysis

\begin{tabular}{|c|c|c|c|c|c|c|c|c|c|}
\hline \multirow{2}{*}{$\begin{array}{l}\text { Dependent } \\
\text { variables }\end{array}$} & \multirow{2}{*}{$\begin{array}{l}\text { Significant independent } \\
\text { variables }\end{array}$} & \multicolumn{4}{|c|}{ US } & \multicolumn{4}{|c|}{ China } \\
\hline & & $\beta$ & t-value & Sig. & $\mathrm{R}^{2}$ & $\beta$ & t-value & Sig. & $\mathrm{R}^{2}$ \\
\hline & & & & & .45 & & & & .338 \\
\hline \multirow{5}{*}{ Renting } & Perceived enjoyment & .343 & 6.100 & .000 & & & & & \\
\hline & Social risk & -.183 & -2.818 & .005 & & -.225 & -2.924 & .004 & \\
\hline & Performance risk & & & & & -.158 & -2.224 & .027 & \\
\hline & Financial risk & & & & & -.164 & -2.141 & .033 & \\
\hline & & & & & .476 & & & & .425 \\
\hline \multirow{5}{*}{ Swapping } & Perceived enjoyment & .313 & 5.655 & .000 & & .272 & 3.937 & .000 & \\
\hline & Social risk & -.285 & -3.986 & .000 & & -.334 & -3.878 & .000 & \\
\hline & Performance risk & & & & & -.182 & -2.363 & .019 & \\
\hline & Psychological risk & & & & & -.232 & -2.986 & .003 & \\
\hline & Financial risk & & & & & -.177 & -2.681 & .008 & \\
\hline
\end{tabular}

Discussion: The results indicated that significant differences exist between American and Chinese consumers in terms of perceived risks and enjoyment of CC as well as attitude toward CC. Furthermore, the study identified that American consumers' intention to rent clothing or to attend swapping events was significantly influenced by perceived enjoyment and social risk; while Chinese consumers' intention to rent clothing was significantly impacted by social risk, performance risk, and financial risk. Differently, Chinese consumers' intention to swap clothing was significantly influenced by perceived enjoyment, social risk, performance risk, psychological risk, and financial risk. 


\section{References}

Botsman, R. \& Rogers, R. (2010). What's Mine is Yours: The Rise of Collaborative Consumption. Harper Business.

Demailly, D. \& Novel, A.-S. (2014). The sharing economy: Make it sustainable. Studies N 03/14, IDDRI, $30 \mathrm{p}$. Retrieved from:

http://www.iddri.org/Evenements/Interventions/ST0314 DD\%20ASN sharing\%20econo $\underline{\text { my.pdf }}$

The Nielsen Global Survey (2014). Is sharing the new buying? Reputation and trust are emerging as new currencies. Retrieved from:

http://www.nielsen.com/content/dam/nielsenglobal/apac/docs/reports/2014/NielsenGlobal-Share-Community-Report.pdf

US EPA. (2014). Textiles common wastes \& materials. Retrieved from: http://www.epa.gov/osw/conserve/materials/textiles.htm

WRAP (2011). Valuing our clothes: The true cost of how we design, use and dispose of clothing in the UK. Retrieved from:

http://www.wrap.org.uk/sites/files/wrap/VoC\%20FINAL \%20online \%202012\%2007\%20 $11 . \mathrm{pdf}$

Xue, H.Y., Zhang, X.J., \& Wang, Y.Q. (2014). Research on the disposal strategy of waste textiles. Applied Mechanics and Materials, 522, 817-820. 